Review

\title{
Survival and innovation: The role of mutational robustness in evolution
}

\author{
Mario A. Fares a, b, * \\ a Instituto de Biología Molecular y Celular de Plantas (CSIC-UPV), C/Ingeniero Fausto Elio, 46022 Valencia, Spain \\ ${ }^{\mathrm{b}}$ Department of Genetics, Smurfit Institute of Genetics, University of Dublin, Trinity College Dublin, Lincoln Place, Dublin 2, Ireland
}

\section{A R T I C L E I N F O}

\section{Article history:}

Received 25 July 2014

Accepted 15 October 2014

Available online $\mathrm{xxx}$

\section{Keywords:}

Mutational robustness

Evolvability

Molecular chaperones

Gene duplication

Fitness landscapes

Functional innovation

\begin{abstract}
A B S T R A C T
Biological systems are resistant to perturbations caused by the environment and by the intrinsic noise of the system. Robustness to mutations is a particular aspect of robustness in which the phenotype is resistant to genotypic variation. Mutational robustness has been linked to the ability of the system to generate heritable genetic variation (a property known as evolvability). It is known that greater robustness leads to increased evolvability. Therefore, mechanisms that increase mutational robustness fuel evolvability. Two such mechanisms, molecular chaperones and gene duplication, have been credited with enormous importance in generating functional diversity through the increase of system's robustness to mutational insults. However, the way in which such mechanisms regulate robustness remains largely uncharacterized. In this review, I provide evidence in support of the role of molecular chaperones and gene duplication in innovation. Specifically, I present evidence that these mechanisms regulate robustness allowing unstable systems to survive long periods of time, and thus they provide opportunity for other mutations to compensate the destabilizing effects of functionally innovative mutations. The findings reported in this study set new questions with regards to the synergy between robustness mechanisms and how this synergy can alter the adaptive landscape of proteins. The ideas proposed in this article set the ground for future research in the understanding of the role of robustness in evolution.
\end{abstract}

(C) 2014 Published by Elsevier B.V.

\section{Introduction}

Biological systems are inherently robust (resistant) to perturbations, maintaining the same phenotype in the face of external challenges and noise internal to the system $[1,2]$. Therefore, phenotypes are said to be robust if they resist perturbations. Robustness was first suggested by Waddington who observed that developmental programs are generally robust to minor perturbations, a property he called canalization $[3,4]$. Robustness applies to many different levels of biological organization and its effects are visible in the expression patterns of genes, resistance of protein structures to mutations and proteins functional promiscuity. The form of robustness most easily measured is the one resulting from the resistance of phenotypes to environmental challenges, such as variable temperatures, limited nutrients and various kinds of environmental stresses. Perturbations also occur within organisms or cells, however less obvious, in the form of variation in expression

\footnotetext{
* Department of Genetics, Smurfit Institute of Genetics, University of Dublin, Trinity College Dublin, Lincoln Place, Dublin 2, Ireland. Tel.: +353 18963521.

E-mail addresses: faresm@tcd.ie, mfares@ibmcp.upv.es.
}

levels and mal-adaptive mutations (e.g., changes in the genetic composition of an individual-also known as genotypic variation). What underlying molecular mechanisms and population genetic parameters provide and regulate the resistance of systems to perturbing mutations remains largely unknown.

The recent meeting in "Protein structure, Protein Evolution" organized by the Royal Swedish Academy of Sciences in June 2014 in Stockholm has provided the ideal scientific environment to link the knowledge generated by structural (phenotype) and evolutionary (genotype) biologists to understand many questions directly or indirectly linked to robustness. The link between the main changes at the structural and sequence levels and the emergence of novel functions has also been discussed and, to a lesser extent, so has the de novo emergence of protein functions. In particular, it has been of great interest the fact that most talks led to distinguishing between the plasticity of protein structures, their tolerance to destabilizing mutations, and the dynamics of the mechanisms of mutational buffering. In this review, I will discuss on the mechanisms of mutational buffering, including gene or genome duplication and heat-shock proteins, as these mechanisms have enthralled researchers for many decades but remain uncharacterized. 
As said before, robustness against environmental and genetic perturbations is a property inherent to all living systems and is ubiquitous to all biological organization levels [5,6]. This tolerance to perturbations, however uncharacterized, have been observed in the form of phenotypic resistance to hundreds of mutations in proteins [7], the resilience of biological networks to single deletions [8], and the resistance of the cell to changes in the expression levels of genes [9]. Moreover, it has been shown that cells are tremendously tolerant to single gene deletions. For example, $80 \%$ of the genes in Saccharomyces cerevisiae growing in lenient laboratory conditions have little to no effect on the fitness of this yeast [10]. Likewise, only $42 \%$ of all the induced gene deletions are essential for mouse viability [11].

The origin and consequences of robustness remain to be uncovered, although a number of observations link robustness to certain biological traits or to the complexity underlying such traits. For example, complex traits seem to be more robust than simple ones to gene perturbations, likely owing to the lower impact of deleterious mutations on the greater set of genes that encode complex traits [12]. The correlation between the complexity of biological traits and the robustness of their encoding genes to

(a)

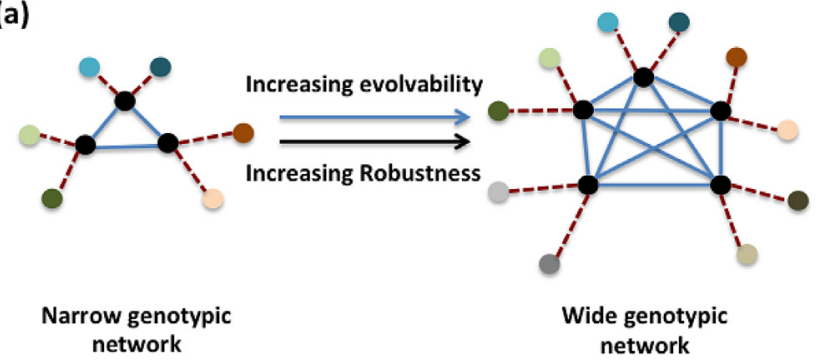

(b)

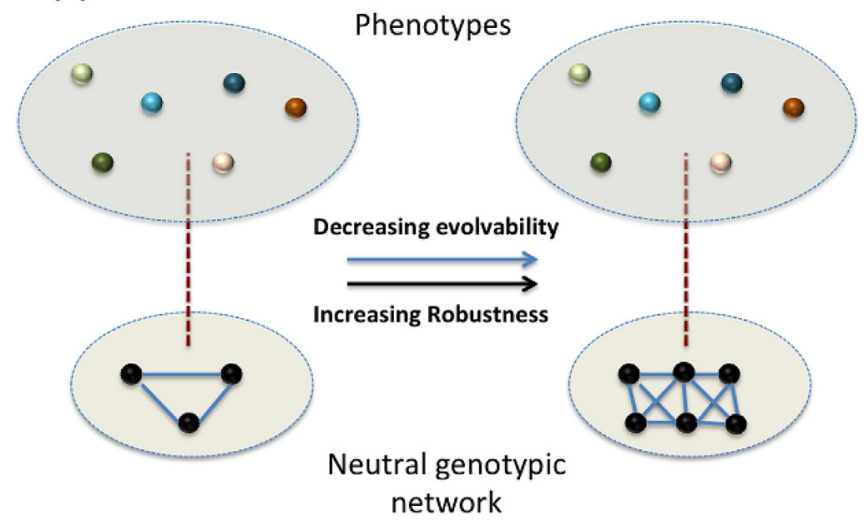

Fig. 1. Increasing robustness leads to larger evolvability and phenotypic plasticity. (a) In this figure I represent two genotypic networks a narrow network and a wide network-a genotypic network is defined as the number of genotypes (black circles) interlinked by a single mutation that are neutral with regards to the phenotype, so that the transition from one genotype to another is phenotypically silent. Increasing the robustness of a biological system increases the genotypic network and the possibility of accessing a larger set of phenotypes (colored circles) through subsequent single mutations. Increasing robustness increases then the evolvability of a system if the set of phenotypes accessible through each genotype by a single mutation is smaller than the entire phenotypic space. In this particular case, the narrow genotypic network in the left of the figure leads to potentially six different accessible phenotypes. Increasing the robustness of the network by two additional genotypes (network in the right of the figure) increases the accessible phenotypic space (evolvability) in four additional phenotypes. (b) This figure represents a case in which each of the genotypes of the same genotypic network can access all of the phenotypes in the phenotypic space. In such a scenario, increasing robustness, that is the number of genotypes in the network, leads to lower evolvability as the number of phenotypes accessible by a single mutation remains constant. mutations suggests a link between robustness and the ability of systems to generate heritable genetic variation (a property known as evolvability). A number of studies have explored the main consequences of increasing robustness and found that there is a complex but significant relationship between robustness and evolvability. Indeed, in a genotypic network, in which the transition between genotypes is phenotypically silent (also known as neutral genotypic network), increasing robustness makes the system more phenotypically evolvable if each of the genotypes can only access a subset of all possible phenotypes [13]. Under this condition, the wider the network the larger is the set of accessible phenotypes, and thus the greater is the system's evolvability (Fig. 1a). Conversely, when each genotype can access all possible phenotypes of the phenotypic space, then higher robustness decreases evolvability (Fig. 1b). Examples on the relationship between robustness and evolvability using entire organisms or populations are lacking in the literature owing to the complexity that experiments devoted to disentangling robustness from other population genetic or environmental factors involves. Advances in the understanding of the role of robustness in evolvability have nevertheless been conducted using simple molecules, in which evolvability can be linked to the distribution of mutations effects on fitness. As a case in point, Hayden and colleagues addressed the role of robustness in
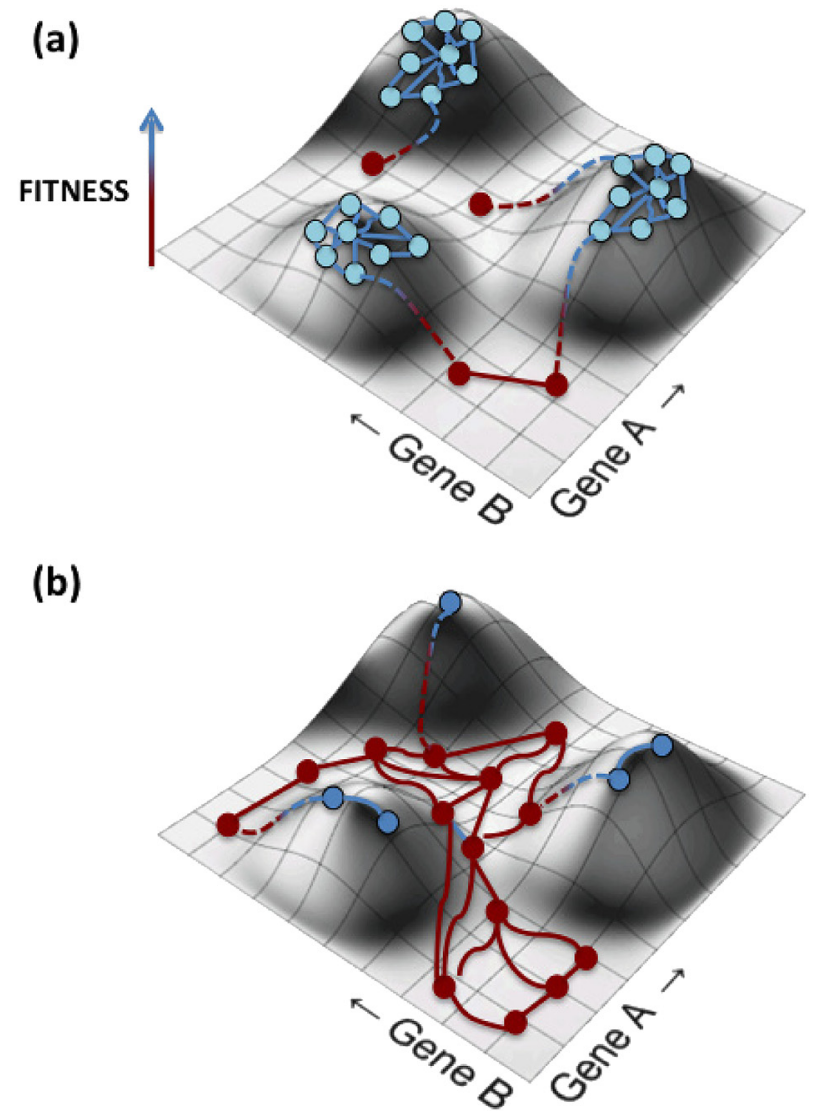

Fig. 2. Distribution of genotypes in a fitness landscape. Circles symbolize genoytpes (red circles are negatively selected genotypes while blue ones are positively selected genotypes) and lines are links between two genotypes, which differ in a single mutation (blue links are allowed transitions by natural selection while red ones are those evolutionary trajectories under strong purifying selection). (a) In a population with high robustness to mutations, most genotypes are part of the same neutral genotypic network and these networks are concentrated on adaptive picks, while genotypes in deleterious valleys are sparsely distributed. (b) Populations with low robustness to mutations present a low number of genotypes populating the adaptive picks with most genotypic transitions being deleterious, and thus leading to genotypes occupying lowfitness valleys in the landscape. 
innovation by evolving a population of RNA enzymes under conditions of neutrality and determining whether such neutral variation can pre-adapt the molecule to other substrates [14]. They showed that, when combined in a single RNA molecule, some genetic variation that was cryptic in the population of RNA enzymes can lead to novel enzymatic capacities when changing environmental conditions-impairment of robustness and the action of natural selection over these previously cryptic variants is known as decanalization [14].

A fundamental condition linking robustness to evolvability is the concept of environmental impairment: robustness leads to the emergence of novel phenotypes if this is impaired by environmental changes. Indeed, copious examples in the literature show that the effect of mutations on fitness is conditional to the genotype-byenvironment interaction, which re-shapes the fitness landscape of a population [15-18]. Fluctuating conditions, including changes in population sizes or in the source of carbon, could change the interaction between mutations (e.g., sign epistasis) and the distribution of mutations fitness effects, allowing population take previously prohibited evolutionary paths $[19,20]$, or precluding them from adopting previously adaptive solutions [21,22]. For example, Flynn and colleagues showed that the positive adaptive interaction of five mutations that were beneficial in Escherichia coli in a specific environment changed to being deleterious when tested in a subset of 1920 environments other than the original one [21].

The relationship between robustness - that is, how wide neutral genotypic networks are in a population-and fitness landscape geometry remains uncharacterized. One could speculate that in a complex fitness landscape containing highly robust populations, the density of genotypes at the adaptive picks should be high, leading to flat adaptive picks, with each of the picks being separated by valleys with sparse genotype distributions (Fig. 2a). Population with low mutational robustness will produce landscapes in

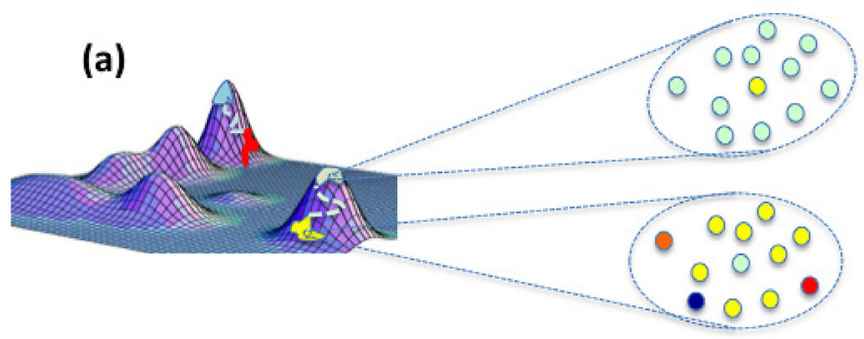

(b)

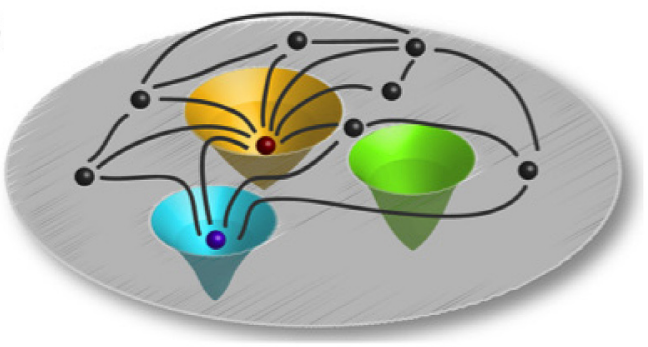

Fig. 3. Navigating fitness landscapes and adaptive traps. Fitness landscapes were first defined by Sewall wright and represent a metaphorical mapping of genotypes to reproductive success. (a) Populations (yellow and red colored forms in the landscape) follow different evolutionary paths (dashed line) in the process of their adaptation to the environment, climbing hills through the selection of the appropriate traits or molecular functions. The genetic reservoir at the top of the hill is substantially lower than that at the bottom of an adaptive hill (colored circles figures emerging from the populations). This decline in variability limits the possibility of adaptive leaps in the landscapes to reach other adaptive hills, entrenching the population into adaptive or fitness traps (b). Escaping such fitness traps requires dynamical landscapes, innovative mutations, epistatic compensatory interactions with newly arising mutations, or increasing robustness of landscapes through mechanisms of mutational robustness. which valleys will be more populated with genotypes and less so will be the adaptive picks, leading to pronounced picks (Fig. 2b). Therefore, the robustness of the population would greatly influence the shape of the picks and genotypic density of the landscape.

While robustness is a well-accepted property, the origins and regulators of mutational robustness remain debated. For example, it is not known whether robustness is an emergent property in every biological system or if it is the result of other selective forces, including those forces imposed by changing environments. Also, it remains unclear how is robustness selected for, what conditions favor it, and what mechanisms regulate it. These points perform the focus of this review, with special emphasis on the role of heatshock proteins (also known as molecular chaperones) and gene or genome duplication in regulating robustness to mutations.

\section{Navigating fitness landscapes silently}

The term of Fitness landscapes was first introduced by Sewall Wright and is routinely used in evolutionary biology to graphically represent the relationship between genotypes and reproductive success (also known as biological fitness) [23]. In these fitness landscapes (Fig. 3a), hills represent regions of optimum fitness while valleys are associated with low fitness. A population of individuals will occupy a particular point of the landscape according to its genotypic composition. If the population occupies a valley, selection will favor genotypes that will bring the population close to nearby adaptive hill, and thus population will always tend to climb adaptive hills dragged by favorable genotypes. The height of the hill is proportional to fitness with higher hills representing larger global fitness. Once a genotype has occupied an adaptive hill (e.g., the population has found a superior phenotype that has become fixed), the population becomes entrenched in what I call adaptive traps (Fig. 3b): At the tip of the hill, the population is genotypically homogeneous and its transition to another adaptive hill is largely halted by its low genetic reservoir and the height of the hill-with compensatory mutations being rare in the population and innovation becoming largely constrained by selection. Populations remain trapped in these adaptive wells unless novel mutations or environmental factors change the surface of the landscape. Increasing population's robustness to mutations, either by reaching highly robust phenotypes or by enhancing mechanisms of robustness or both these possibilities, will smooth the abruptness of the fitness landscape enabling the neutral transition of populations to other adaptive hills. In the limit, intermediate levels of robustness would bridge different adaptive hills thereby enabling large evolutionary leaps. In the remainder of this review I will focus on two main mechanisms of mutational robustness, heat-shock proteins and gene duplication, which have been credited with an important role in biological innovation but their regulatory mechanism of robustness to either environmental or genetic perturbations is yet to be characterized.

\section{Molecular chaperones capacitate novel adaptations}

The Heat shock proteins (also known as molecular chaperones) are essential to the cell in normal physiological conditions [24-27] but more so under stress, allowing cells and organisms survive otherwise lethal conditions [25,28,29]. Molecular chaperones perform a bewildering set of essential functions in the cell, including folding, trafficking, and degradation of other proteins [30]. Chief among all the functions performed by chaperones is protein folding, an essential step in the performance of proteins functions. Protein folding is particularly relevant for slow-folding proteins, generally also those that are big proteins, which are prone to form non-specific aggregates when misfolded [31]. 
Chaperones therefore supervise the proper function of proteins by folding de novo or re-folding proteins after stress, preventing the promiscuous associations between non-native polypeptides [32].

As much as temperature is a force in leading to misaggregated proteins, mutations also destabilize proteins if unsupervised owing to their stochastic origin. Chaperones have been reported as buffering machines of the destabilizing effects of mutations as they fold mutated versions of proteins into their productive conformation, maintaining mutations cryptic in the population for long periods of time [33-36]. When the buffering mechanism of chaperones is impaired, mutations can reach such levels in the populations that, when combined in a single genome, they could lead to adaptations to previously unexplored environments. Therefore, chaperones have been considered as capacitors of evolution. Two main chaperones have been extensively explored for their ability to buffer the effects of mutations, Hsp90 and GroEL. In the following sections I provide evidence that such chaperones are enhancers of the mutational robustness of biological systems and capacitors of evolution.

\subsection{Hsp90 canalizes and capacitates morphological variation in eukaryotes}

Hsp90 is an essential molecular chaperone that assists in the folding of proteins whose structure stability is key to their functions, including steroid hormone receptors, kinases, transcription factors, and ubiquitin ligases. Hsp90 is generally synthesized at levels that are higher than needed for the folding activity, thereby maintaining these proteins stable and avoiding the otherwise deleterious phenotypic variants. It was therefore suspected that environmental perturbations that compromise the folding assistance provided by Hsp90 would unfold a cascade of phenotypic changes, many of which, however non-adaptive under normal physiological conditions, may combine in the genome in such a way that would become adaptive under other conditions, even after restoring the levels of Hsp90. In agreement with this prediction, Lindquist and colleagues have demonstrated in a number of recent studies that Hsp90 can mask the phenotypic effects of mutations, maintaining genetic variation cryptic in the population [36-42], a phenomenon supporting the concept of "canalization" that was proposed by Conrad Waddington [3]. When the buffering mechanism of Hsp90 is impaired, either through high temperatures or pharmacologically through Hsp90-specific inhibitors such as Geldanamycin or Radicicol, the previously cryptic genetic variation is expressed and selection can then act upon it, a phenomenon known as "decanalization" [36,39].

While a number of laboratory experiments in model organisms have demonstrated the buffering capacity of Hsp90, the link between this buffering and the emergence of adaptive traits has remained controversial. In a very recent experiment, however, Rohner and colleagues have shown that the buffering of genetic variation by Hsp90 is the basis for the adaptation of surface populations of the cavefish Astyanax mexicanus to cave environments [40]. Indeed, they showed that cave environments can compromise Hsp90 capacity releasing eye morphological variation to the same extent as geldanamycin does with surface river populations of this fish. Therefore, their study demonstrated in a very elegant and direct way that Hsp90 has contributed to reducing eye size in cave conditions, where the development of normal eye size may tax other most needed sensory mechanisms, and thus Hsp90 acted as a capacitor of adaptive morphological variation in a natural setting.

An expected outcome of the Hsp90 buffering capacity is that proteins requiring Hsp90 for folding, such as kinases, transcription factors and signal transducing molecules should tolerate more mutations and evolve faster than those proteins not requiring Hsp90 for folding under the same selective pressures. Accordingly, duplicated genes in yeast in which one of the gene copies, but not its paralog, is a client of Hsp90 shows faster evolution at the Hsp90 client copy than its non-client paralog [43]. Moreover, one of the two paralogs of a transcription factor in the brassinosteroid pathway in the plant Arabidopsis thaliana that requires Hsp90 for folding shows more relaxed constraints than its non-client paralog [43]. Since duplicates are considered identical or nearly so at their birth, such results are not artifacts caused by the differential protein stabilities. The finding in another recent study that strong and weak substrate of Hsp90 do not evolve faster than non-clients but they do show larger fraction of non-conservative, radical, amino acid substitutions, sparks the idea that a complex relationship exists between the buffering ability of chaperones and the structural features of protein clients [44]. This relationship and the factors governing it require further investigation, which is currently being conducted by a number of leading laboratories.

\subsection{GroEL provides robustness to mutations and facilitates the emergence of alternative promiscuous enzymatic functions}

GroEL, and its cohcaperonin GroES, belong to an evolutionarily conserved family of heat-shock proteins that perform essential functions in the cell. Chief among these functions is their noncovalent interaction with partially folded proteins (also known as client proteins) to prevent their non-specific aggregation and help in their proper folding [45-47]. GroEL has been found to be essential for $E$. coli at a broad range of temperatures and not only under heat stress [48]. The genes encoding GroEL and GroES are part of the same operon ( $g r o E$ ) which is ubiquitous in all prokaryotes and eukaryotic organelles, although they have also been found in archaea [49].

GroEL has been found to facilitate the folding of proteins, and their mutated versions, into their final productive conformation. Their modulation of the relationship between sequence and structure-that is between genotype and phenotype-has suggested their role as phenotypic capacitors. Indeed, Nancy Moran suggested that GroEL might buffer the destabilizing effects of mutations in the proteins of endosymbiotic bacteria of insects, known for their drifting evolutionary dynamics [35]. Endosymbiotic bacteria of insects, as many pathogenic bacteria, are maternally inherited and transmitted to the next generations in small numbers (e.g., the populations are subjected to large bottlenecks between generations) [50], and thus genetic drift plays a more important role than selection in their evolutionary dynamics [35]. This stronger genetic drift in endosymbiotic bacteria leads to the fixation of deleterious or slightly deleterious mutations. Moran proposed that GroEL might buffer the deleterious effects of mutations by properly folding mutated protein versions. This hypothesis is supported by the overproduction of GroEL in all known endosymbiotic mutualistic bacteria of insects but not in free-living bacteria [51-53]. Despite the evolutionary conservation of GroEL sequence in endosymbiotic bacteria, fixation of adaptive mutations in GroEL regions important for binding and folding protein clients supports the role of positive selection in increasing the folding ability of endosymbiotic GroEL compared to its free-living homolog [54].

Fares and colleagues provided the first evidence in support of the GroEL buffering capacity, by showing that over-expression of groE in $E$. coli strains that evolved under strong genetic drift for thousands of $E$. coli generations could rescue cells with declined fitness [34]. The main hypothesis derived from this study was that GroEL was directly able to buffer the effects of deleterious destabilizing mutations. This evidence was supported by a later study in which authors showed that mutated versions of the operon groE with increased expression were recurrently emerging in drifting evolutionary lineages of Salmonella typhimurium and that this over- 
expression mitigates the deleterious effects of random mutations through antagonistic epistasis [55]. The relationship between GroEL over-production and the fixation of deleterious mutations, however unknown, has been shown to be complex. Some evidence pinpoints the function of GroEL in concert with other protein quality control mechanisms, such as the protease Lon, in the folding of proteins at intermediate states of folding, thereby maintaining an equilibrium of folded and active proteins in the cell [56]. Overexpression of groE has been also shown to rescue the fitness of cells bearing a variety of slow-growing, or even deleterious, DHFR mutants and restore their growth to wild type levels [56]. A prediction derived from these studies is that protein clients requiring GroEL for folding should be candidates for accelerated evolution more readily so than proteins those do not require GroEL. Indeed, GroEL clients have been shown to evolve at a faster rate than nonclient proteins (e.g., they fix more amino acid replacing mutations), once gene essentiality, protein-protein interaction degree and expression levels-known factors to influence the rates of evolution [57] - have been controlled for [58,59].

Despite evidence supporting the role of GroEL in mitigating the deleterious effects of destabilizing mutations, the link between this buffering ability and the emergence of evolutionary novelties through mutational robustness remains missing. Recent experiments have shed some light on the direct role of GroEL in evolutionary innovation. In their study, Tokuriki and Tawfik demonstrated that over-expression of groE doubles the number of mutations tolerated by an enzyme owing to the GroEL increased folding capacity of enzymes carrying highly destabilizing mutations [60]. This increased stability of mutated enzyme versions allows alternative promiscuous functions overcome their stability trade-offs and substantially increase their activity and specificity for substrates other then their preferred one [60,61]. Whether GroEL stabilizes structurally compromised protein versions for long enough to provide an opportunity for subsequent mutations to compensate such destabilizing effects is not known. However, these mechanisms may be crucial in relieving GroEL from its compensatory role in genomes highly loaded with deleterious mutations, such as those belonging to ancient endosymbiotic bacteria of insects. The synergy between GroEL and other compensatory mechanisms in ameliorating the effects of mutations remains obscure and will be an arena for future research.

\section{Evolution by gene duplication: robustness to mutational insults}

Because of its ability to generate novel genes and functions, gene duplication has been credited with enormous evolutionary importance [62]. The contribution of gene duplication to the emergence of novel functions is understood in the context of generating novel genetic material. After the duplication of a gene, the two virtually identical copies of the ancestral gene share identical set of functions (although see Ref. [63]). The naïve classic view is that after duplication, while one of the copies performs the ancestral functions, the other, freed from selective constraints, explores a wide range of alternative phenotypes. Inherent to this theory is the assumption that such alternative phenotypes are often in the vicinity of the genotypic networks of duplicated genes, such that a single amino acid change in the protein encoded by one of the gene copies should provide access to these phenotypic neighborhood. More realistic and complex evolutionary models have been devised (see for review [64]). The link between gene duplication, and more specifically genome duplication, and biological innovation has been established in a number of studies based on evidence from comparative genomics and fossil record. Accordingly, the concomitant emergence of protein families through genome duplication and Angiosperms morphological and physiological explosive diversification during the cretaceous era [65-74] and, although less frequent, with the morphological diversification of vertebrates $[75,76]$ led researchers to causally link duplication with innovation. The mechanism of duplication, that is whole-genome vs small-scale duplications, has been also shown to differentially contribute to the emergence of innovations [77], with the former contributing more to subfunctionalization (e.g., the partition of ancestral functions between the two daughter gene copies) and the latter to the emergence of independent functions [78].

Models concerned with the evolution of duplicated genes differ in their philosophical assumptions with regards to the role of natural selection in facilitating the emergence of novel functions through genetic redundancy. In particular, whether mutational robustness caused by genetic redundancy in duplicated genes is selected upon remains the subject of intense debate. While gene duplicates have been considered to have a major role in mutational robustness through genetic redundancy [79], the persistence of genes in duplicate clashes with the evolutionary instability of genetic redundancy. Nonetheless, evidence support a role of duplicated genes in mutational robustness: a) The deletion of singletons in yeast have larger fitness effects than the deletion of duplicates [80]; b) Deletion of gene copies in yeast are often functionally compensated by the other gene copy, thereby having lower effects on genetic interactions for duplicates than singletons [81]; c) Duplicates present higher robustness than singletons to transient gene knock-downs in Caenorhabditis elegans [82], and d) Duplicates contribute to back-up against deleterious mutations in humans [83]. Against this evidence, using synthetic lethality genetic maps it has been shown that duplicates only account for $25 \%$ of the genetic robustness of yeast [84]. Moreover, in natural populations close duplicates are unlikely to provide mutational robustness [85]. Therefore, whether or not gene duplication provides robustness to mutations remains under question.

A gene duplication can facilitate innovation of biological functions through two main steps: a) increasing the resilience of phenotypes encoded by duplicates to mutational changes (e.g., genotypic diversity does not cause visible phenotypic diversity in robust systems), and thus generating genotypic diversity that remains silent in the population and b) by canalizing genotypic diversity such that it facilitates the emergence of novel phenotypes once robustness is impaired. Support to the generation of neutral genotypic diversity after gene duplication comes from the classic belief that after duplication gene copies suffer relaxed selective constraints that allow one or both copies tolerating many-fold more mutations than otherwise, a fact with extensive experimental and theoretical support $[2,86,87]$. This phenomenon is more obvious when analyzing genomes populated with duplicates originated by whole-genome duplication, in which younger duplicates still preserve signatures of their larger tolerance to mutations than older duplicates $[79,84,88,89]$. The fact that expansion of certain protein families is concomitant with the emergence of morphological diversity sparks the possibility that gene duplication is linked to robustness and evolvability. A remarkable example of this link is the expansion of the MADS box proteins in plants and the diversity in plants flowering. This protein family, although ubiquitous in extant eukaryotes, it has undergone a wave of duplication events in the ancestor of flowering plants [90-93]. The almost total functional overlap in the proteins functions between MADS box duplicates, such as the 4 copies of SEPALLATA genes responsible of the formation of reproductive organs in flowering plants [94-96] attests to the important role that such duplicates played in robustness to mutations. These duplicates have, however, diversified in other species probably resulting from impairment of robustness by environmental challenges [97]. 
Another question that rises is how does the cell cope with the increase gene dosage resulting from gene duplication, particularly when such duplication affects only a fraction of the genes [98-100]. Evidence suggests that such genes diverge in expression so that they perform similar functions under different stress conditions and that the expression of genes in aneuploidy are more robust to changes than those in single copies [101].

A key point in the understanding of the link between gene duplication and mutational robustness is the mechanism by which mutational robustness is favored by natural selection. Certainly robustness to mutations cannot be a trait selected favorably because it provides genetic variation and potential for evolvability, as a central tenet of Darwinian evolution is that evolution is not a forward-looking process. Notwithstanding the fact that understanding the link between robustness and selection requires complex mathematical modeling, one can imagine that a population that acquires evolvability as a trait has higher probability of acquiring beneficial mutations and producing offspring with increased fitness. In a scenario in which evolvability, produced by higher robustness, persists long enough in the population as to increase the frequency of individuals with such trait, this trait would be selected favorably because it increases the chances for beneficial mutations to appear in the population [102], a process known as genetic hitchhiking [103]. In the case of duplicated genes, these are likely to return to single gene copies right after duplication, unless they remain in the genome because they increase the mutational robustness of the gene and the evolvability of populations in terms of the emergence of new functions and regulations [104]. Such robustness is likely to be selected positively if the environment is rapidly changing so that robustness facilitate the persistence of two gene copies and increase the possibility for the divergence between the sister gene copies at the regulatory as well as the functional levels, finally resulting in the emergence of adaptations to the new environments $[78,104]$.

\section{Concluding remarks}

Mutational robustness is a property inherent to all known biological systems and has been widely regarded as key to evolvability. Mechanisms regulating robustness are crucial to canalize genetic variation and facilitate the emergence of biological innovations, enabling major evolutionary leaps. Molecular chaperones and Gene duplication have enthralled researchers for decades because of their main role in impinging robustness to genes and fueling innovation. A broad range of studies and observations support that both these two mechanisms have been paramount to regulate the emergence of major innovations, driving the main events of biological diversification and the origin of complexity on earth.

\section{Conflict of interest}

The author declares that there is no conflict of interest involved in this study.

\section{Acknowledgments}

This study was supported by Science Foundation Ireland (grant reference: $12 / \mathrm{IP} / 1673$ ) and a grant from the Spanish Ministerio de Economía y Competitividad (BFU2009-12022) to MAF.

\section{References}

[1] A. Wagner, Robustness, evolvability, and neutrality, FEBS Lett. 579 (2005) $1772-1778$.
[2] A. Wagner, The role of robustness in phenotypic adaptation and innovation, Proc. R. Soc. Biol. Sci. 279 (2012) 1249-1258.

[3] C.H. Waddington, Canalization of development and the inheritance of required characters, Nature 150 (1942) 3.

[4] C.H. Waddington, Genetic assimilation of an acquired character, Evol. Int. J. Org. Evol. 7 (1953) 9.

[5] J.A. de Visser, J. Hermisson, G.P. Wagner, L. Ancel Meyers, H. Bagheri-Chaichian, J.L. Blanchard, L. Chao, J.M. Cheverud, S.F. Elena, W. Fontana, G. Gibson, T.F. Hansen, D. Krakauer, R.C. Lewontin, C. Ofria, S.H. Rice, G. von Dassow, A. Wagner, M.C. Whitlock, Perspective: evolution and detection of genetic robustness, Evol. Int. J. Org. Evol. 57 (2003) 1959-1972.

[6] A. Wagner, Robustness and Evolvability in Living Systems, Princeton University Press, New Jersey, 2005.

[7] N. Sinha, R. Nussinov, Point mutations and sequence variability in proteins redistributions of preexisting populations, Proc. Natl. Acad. Sci. U. S. A. 98 (2001) 3139-3144.

[8] M. Aldana, E. Balleza, S. Kauffman, O. Resendiz, Robustness and evolvability in genetic regulatory networks, J. Theor. Biol. 245 (2007) 433-448.

[9] N.N. Batada, L.D. Hurst, Evolution of chromosome organization driven by selection for reduced gene expression noise, Nat. Genet. 39 (2007) 945-949.

[10] G. Giaever, A.M. Chu, L. Ni, C. Connelly, L. Riles, S. Veronneau, S. Dow, A. Lucau-Danila, K. Anderson, B. Andre, A.P. Arkin, A. Astromoff, M. El-Bakkoury, R. Bangham, R. Benito, S. Brachat, S. Campanaro, M. Curtiss, K. Davis, A. Deutschbauer, K.D. Entian, P. Flaherty, F. Foury, D.J. Garfinkel, M. Gerstein, D. Gotte, U. Guldener, J.H. Hegemann, S. Hempel, Z. Herman, D.F. Jaramillo, D.E. Kelly, S.L. Kelly, P. Kotter, D. LaBonte, D.C. Lamb, N. Lan, H. Liang, H. Liao, L. Liu, C. Luo, M. Lussier, R. Mao, P. Menard, S.L. Ooi, J.L. Revuelta, C.J. Roberts, M. Rose, P. Ross-Macdonald, B. Scherens, G. Schimmack, B. Shafer, D.D. Shoemaker, S. Sookhai-Mahadeo, R.K. Storms, J.N. Strathern, G. Valle, M. Voet, G. Volckaert, C.Y. Wang, T.R. Ward, J. Wilhelmy, E.A. Winzeler, Y. Yang, G. Yen, E. Youngman, K. Yu, H. Bussey, J.D. Boeke, M. Snyder, P. Philippsen, R.W. Davis, M. Johnston, Functional profiling of the Saccharomyces cerevisiae genome, Nature 418 (2002) 387-391.

[11] J.K. White, A.K. Gerdin, N.A. Karp, E. Ryder, M. Buljan, J.N. Bussell, J. Salisbury S. Clare, N.J. Ingham, C. Podrini, R. Houghton, J. Estabel, J.R. Bottomley, D.G. Melvin, D. Sunter, N.C. Adams, P. Sanger Institute Mouse Genetics, D. Tannahill, D.W. Logan, D.G. Macarthur, J. Flint, V.B. Mahajan, S.H. Tsang I. Smyth, F.M. Watt, W.C. Skarnes, G. Dougan, D.J. Adams, R. Ramirez-Solis, A. Bradley, K.P. Steel, Genome-wide generation and systematic phenotyping of knockout mice reveals new roles for many genes, Cell 154 (2013) 452-464.

[12] W.C. Ho, J. Zhang, The genotype-phenotype map of yeast complex traits: basic parameters and the role of natural selection, Mol. Biol. Evol. 31 (2014) $1568-1580$.

[13] J.A. Draghi, T.L. Parsons, G.P. Wagner, J.B. Plotkin, Mutational robustness can facilitate adaptation, Nature 463 (2010) 353-355.

[14] E.J. Hayden, E. Ferrada, A. Wagner, Cryptic genetic variation promotes rapid evolutionary adaptation in an RNA enzyme, Nature 474 (2011) 92-95.

[15] S. Alizon, M. van Baalen, Emergence of a convex trade-off between trans mission and virulence, Am. Nat. 165 (2005) E155-E167.

[16] S.C. Kim, L.H. Rieseberg, The contribution of epistasis to species differences in annual sunflowers, Mol. Ecol. 10 (2001) 683-690.

[17] R. Kishony, S. Leibler, Environmental stresses can alleviate the average deleterious effect of mutations, J. Biol. 2 (2003) 14.

[18] S.K. Remold, R.E. Lenski, Pervasive joint influence of epistasis and plasticity on mutational effects in Escherichia coli, Nat. Genet. 36 (2004) 423-426.

[19] J.A. Coyne, N.H. Barton, M. Turelli, Is Wright's shifting balance process important in evolution? Evolution 54 (2000) 306-317.

[20] M.J. Wade, J.R. Griesemer, Populational heritability: empirical studies of evolution in metapopulations, Am. Nat. 151 (1998) 135-147.

[21] K.M. Flynn, T.F. Cooper, F.B. Moore, V.S. Cooper, The environment affects epistatic interactions to alter the topology of an empirical fitness landscape, PLoS Genet. 9 (2013) e1003426.

[22] A.R. Hall, Genotype-by-environment interactions due to antibiotic resistance and adaptation in Escherichia coli, J. Evol. Biol. 26 (2013) 1655-1664.

[23] S. Wright, The roles of mutation, inbreeding, crossbreeding, and selection in evolution, in: Proceedings of the Sixth International Congress on Genetics, 1932, pp. 355-366.

[24] B. Bukau, A.L. Horwich, The Hsp70 and Hsp60 chaperone machines, Cell 92 (1998) 351-366

[25] F.U. Hartl, M. Hayer-Hartl, Molecular chaperones in the cytosol: from nascent chain to folded protein, Science 295 (2002) 1852-1858.

[26] T. Hesterkamp, B. Bukau, Role of the DnaK and HscA homologs of Hsp70 chaperones in protein folding in E. coli, EMBO J. 17 (1998) 4818-4828.

[27] M.P. Mayer, B. Bukau, Hsp70 chaperone systems: diversity of cellular functions and mechanism of action, Biol. Chem. 379 (1998) 261-268.

[28] M.E. Feder, G.E. Hofmann, Heat-shock proteins, molecular chaperones, and the stress response: evolutionary and ecological physiology, Annu. Rev. Physiol. 61 (1999) 243-282.

[29] H. Sakahira, P. Breuer, M.K. Hayer-Hartl, F.U. Hartl, Molecular chaperones as modulators of polyglutamine protein aggregation and toxicity, Proc. Natl. Acad. Sci. U. S. A. 99 (Suppl. 4) (2002) 16412-16418.

[30] L.E. Hightower, Heat shock, stress proteins, chaperones, and proteotoxicity, Cell 66 (1991) 191-197. 
[31] J. Buchner, Supervising the fold: functional principles of molecular chaperones, FASEB J. Off. Publ. Fed. Am. Soc. Exp. Biol. 10 (1996) 10-19.

[32] S.L. Rutherford, Between genotype and phenotype: protein chaperones and evolvability, Nat. Rev. Genet. 4 (2003) 263-274.

[33] M.A. Fares, A. Moya, E. Barrio, GroEL and the maintenance of bacterial endosymbiosis, Trends Genet. 20 (2004) 413-416.

[34] M.A. Fares, M.X. Ruiz-Gonzalez, A. Moya, S.F. Elena, E. Barrio, Endosymbiotic bacteria: groEL buffers against deleterious mutations, Nature 417 (2002) 398

[35] N.A. Moran, Accelerated evolution and Muller's rachet in endosymbiotic bacteria, Proc. Natl. Acad. Sci. U. S. A. 93 (1996) 2873-2878.

[36] S.L. Rutherford, S. Lindquist, Hsp90 as a capacitor for morphological evolution, Nature 396 (1998) 336-342.

[37] L.E. Cowen, S. Lindquist, Hsp90 potentiates the rapid evolution of new traits: drug resistance in diverse fungi, Science 309 (2005) 2185-2189.

[38] D.F. Jarosz, S. Lindquist, Hsp90 and environmental stress transform the adaptive value of natural genetic variation, Science 330 (2010) 1820-1824.

[39] C. Queitsch, T.A. Sangster, S. Lindquist, Hsp90 as a capacitor of phenotypic variation, Nature 417 (2002) 618-624.

[40] N. Rohner, D.F. Jarosz, J.E. Kowalko, M. Yoshizawa, W.R. Jeffery R.L. Borowsky, S. Lindquist, C.J. Tabin, Cryptic variation in morphologica evolution: HSP90 as a capacitor for loss of eyes in cavefish, Science 342 (2013) 1372-1375.

[41] M. Taipale, I. Krykbaeva, M. Koeva, C. Kayatekin, K.D. Westover, G.I. Karras, S. Lindquist, Quantitative analysis of HSP90-client interactions reveals principles of substrate recognition, Cell 150 (2012) 987-1001.

[42] Y. Xu, M.A. Singer, S. Lindquist, Maturation of the tyrosine kinase c-src as a kinase and as a substrate depends on the molecular chaperone Hsp90, Proc Natl. Acad. Sci. U. S. A. 96 (1999) 109-114.

[43] J. Lachowiec, T. Lemus, J.H. Thomas, P.J. Murphy, J.L. Nemhauser, C. Queitsch, The protein chaperone HSP90 can facilitate the divergence of gene duplicates, Genetics 193 (2013) 1269-1277.

[44] S. Pechmann, J. Frydman, Interplay between chaperones and protein disorder promotes the evolution of protein networks, PLoS Comput. Biol. 10 (2014) e1003674.

[45] W.A. Fenton, A.L. Horwich, GroEL-mediated protein folding, Protein Sci. 6 (1997) 743-760

[46] Z. Lin, H.S. Rye, GroEL-mediated protein folding: making the impossible, possible, Crit. Rev. Biochem. Mol. Biol. 41 (2006) 211-239.

[47] F.U. Hartl, M. Hayer-Hartl, Converging concepts of protein folding in vitro and in vivo, Nat. Struct. Mol. Biol. 16 (2009) 574-581.

[48] O. Fayet, T. Ziegelhoffer, C. Georgopoulos, The groES and groEL heat shock gene products of Escherichia coli are essential for bacterial growth at all temperatures, J. Bacteriol. 171 (1989) 1379-1385.

[49] T.A. Williams, F.M. Codoner, C. Toft, M.A. Fares, Two chaperonin systems in bacterial genomes with distinct ecological roles, Trends Genet. 26 (2010) 47-51.

[50] C. Braendle, T. Miura, R. Bickel, A.W. Shingleton, S. Kambhampati, D.L. Stern, Developmental origin and evolution of bacteriocytes in the aphid-Buchnera symbiosis, PLoS Biol. 1 (2003) E21.

[51] S. Aksoy, Molecular analysis of the endosymbionts of tsetse flies: 16S rDNA locus and over-expression of a chaperonin, Insect Mol. Biol. 4 (1995) 23-29.

[52] H. Charles, A. Heddi, J. Guillaud, C. Nardon, P. Nardon, A molecular aspect of symbiotic interactions between the weevil Sitophilus oryzae and its endosymbiotic bacteria: over-expression of a chaperonin, Biochem. Biophys. Res. Commun. 239 (1997) 769-774.

[53] S. Sato, H. Ishikawa, Expression and control of an operon from an intracellular symbiont which is homologous to the groE operon, J. Bacteriol. 179 (1997) 2300-2304.

[54] M.A. Fares, E. Barrio, B. Sabater-Munoz, A. Moya, The evolution of the heatshock protein GroEL from Buchnera, the primary endosymbiont of aphids, is governed by positive selection, Mol. Biol. Evol. 19 (2002) 1162-1170.

[55] S. Maisnier-Patin, J.R. Roth, A. Fredriksson, T. Nystrom, O.G. Berg D.I. Andersson, Genomic buffering mitigates the effects of deleterious mutations in bacteria, Nat. Genet. 37 (2005) 1376-1379.

[56] S. Bershtein, W. Mu, A.W. Serohijos, J. Zhou, E.I. Shakhnovich, Protein quality control acts on folding intermediates to shape the effects of mutations on organismal fitness, Mol. Cell. 49 (2013) 133-144.

[57] D.A. Drummond, C.O. Wilke, Mistranslation-induced protein misfolding as dominant constraint on coding-sequence evolution, Cell 134 (2008) $341-352$.

[58] D. Bogumil, T. Dagan, Chaperonin-dependent accelerated substitution rates in prokaryotes, Genome Biol. Evol. 2 (2010) 602-608.

[59] T.A. Williams, M.A. Fares, The effect of chaperonin buffering on protein evolution, Genome Biol. Evol. 2 (2010) 609-619.

[60] N. Tokuriki, D.S. Tawfik, Chaperonin overexpression promotes genetic variation and enzyme evolution, Nature 459 (2009) 668-673.

[61] M. Soskine, D.S. Tawfik, Mutational effects and the evolution of new protein functions, Nat. Rev. Genet. 11 (2010) 572-582.

[62] S. Ohno, Evolution by Gene Duplication, Springer-Verlag, Berlin, 1970.

[63] M. Lynch, V. Katju, The altered evolutionary trajectories of gene duplicates, Trends Genet. 20 (2004) 544-549.

[64] H. Innan, F. Kondrashov, The evolution of gene duplications: classifying and distinguishing between models, Nat. Rev. Genet. 11 (2010) 97-108.

[65] G. Blanc, K.H. Wolfe, Functional divergence of duplicated genes formed by polyploidy during Arabidopsis evolution, Plant cell 16 (2004) 1679-1691.
[66] J.F. Wendel, Genome evolution in polyploids, Plant Mol. Biol. 42 (2000) $225-249$

[67] J.S. Taylor, J. Raes, Duplication and divergence: the evolution of new genes and old ideas, Annu. Rev. Genet. 38 (2004) 615-643.

[68] O. Lespinet, Y.I. Wolf, E.V. Koonin, L. Aravind, The role of lineage-specific gene family expansion in the evolution of eukaryotes, Genome Res. 12 (2002) 1048-1059.

[69] E.B. Holub, The arms race is ancient history in Arabidopsis, the wildflower, Nat. Rev. Genet. 2 (2001) 516-527.

[70] S. Maere, S. De Bodt, J. Raes, T. Casneuf, M. Van Montagu, M. Kuiper, Y. Van de Peer, Modeling gene and genome duplications in eukaryotes, Proc. Natl. Acad. Sci. U. S. A. 102 (2005) 5454-5459.

[71] S. Aoki, K. Uehara, M. Imafuku, M. Hasebe, M. Ito, Phylogeny and divergence of basal angiosperms inferred from APETALA3- and PISTILLATA-like MADSbox genes, J. Plant Res. 117 (2004) 229-244.

[72] S. Kim, M.J. Yoo, V.A. Albert, J.S. Farris, P.S. Soltis, D.E. Soltis, Phylogeny and diversification of B-function MADS-box genes in angiosperms: evolutionary and functional implications of a 260-million-year-old duplication, Am. J. Bot. 91 (2004) 2102-2118.

[73] E.M. Kramer, R.L. Dorit, V.F. Irish, Molecular evolution of genes controlling petal and stamen development: duplication and divergence within the APETALA3 and PISTILLATA MADS-box gene lineages, Genetics 149 (1998) $765-783$

[74] M.D. Purugganan, S.D. Rounsley, R.J. Schmidt, M.F. Yanofsky, Molecular evolution of flower development: diversification of the plant MADS-box regulatory gene family, Genetics 140 (1995) 345-356.

[75] S. Hoegg, H. Brinkmann, J.S. Taylor, A. Meyer, Phylogenetic timing of the fishspecific genome duplication correlates with the diversification of teleost fish, J. Mol. Evol. 59 (2004) 190-203.

[76] S.P. Otto, J. Whitton, Polyploid incidence and evolution, Annu. Rev. Genet. 34 (2000) 401-437.

[77] L. Carretero-Paulet, M.A. Fares, Evolutionary dynamics and functional specialization of plant paralogs formed by whole and small-scale genome duplications, Mol. Biol. Evol. 29 (2012) 3541-3551.

[78] M.A. Fares, O.M. Keane, C. Toft, L. Carretero-Paulet, G.W. Jones, The roles of whole-genome and small-scale duplications in the functional specialization of Saccharomyces cerevisiae genes, PLoS Genet. 9 (2013) e1003176.

[79] M. Lynch, J.S. Conery, The evolutionary fate and consequences of duplicate genes, Science 290 (2000) 1151-1155.

[80] Z. Gu, L.M. Steinmetz, X. Gu, C. Scharfe, R.W. Davis, W.H. Li, Role of duplicate genes in genetic robustness against null mutations, Nature 421 (2003) 63-66.

[81] B. VanderSluis, J. Bellay, G. Musso, M. Costanzo, B. Papp, F.J. Vizeacoumar, A. Baryshnikova, B. Andrews, C Boone, C.L Myers, Genetic interactions reveal the evolutionary trajectories of duplicate genes, Mol. Syst. Biol. 6 (2010) 429

[82] G.C. Conant, A. Wagner, Duplicate genes and robustness to transient gene knock-downs in Caenorhabditis elegans, Proc. R. Soc. Biol. Sci. 271 (2004) 89-96.

[83] T.L. Hsiao, D. Vitkup, Role of duplicate genes in robustness against deleterious human mutations, PLoS Genet. 4 (2008) e1000014.

[84] J. Ihmels, S.R. Collins, M. Schuldiner, N.J. Krogan, J.S. Weissman, Backup without redundancy: genetic interactions reveal the cost of duplicate gene loss, Mol. Syst. Biol. 3 (2007) 86.

[85] G. Plata, D. Vitkup, Genetic robustness and functional evolution of gene duplicates, Nucleic Acids Res. 42 (2014) 2405-2414.

[86] A. Wagner, Distributed robustness versus redundancy as causes of mutational robustness, BioEssays News Rev. Mol. Cell. Dev. Biol. 27 (2005) $176-188$

[87] A. Wagner, Gene duplications, robustness and evolutionary innovations, BioEssays News Rev. Mol. Cell. Dev. Biol. 30 (2008) 367-373.

[88] Z. Gu, A. Cavalcanti, F.C. Chen, P. Bouman, W.H. Li, Extent of gene duplication in the genomes of Drosophila, nematode, and yeast, Mol. Biol. Evol. 19 (2002) $256-262$.

[89] R. Harrison, B. Papp, C. Pal, S.G. Oliver, D. Delneri, Plasticity of genetic interactions in metabolic networks of yeast, Proc. Natl. Acad. Sci. U. S. A. 104 (2007) 2307-2312.

[90] G. Theissen, A. Becker, A. Di Rosa, A. Kanno, J.T. Kim, T. Munster, K.U. Winter, H. Saedler, A short history of MADS-box genes in plants, Plant Mol. Biol. 42 (2000) 115-149.

[91] E.R. Alvarez-Buylla, S.J. Liljegren, S. Pelaz, S.E. Gold, C. Burgeff, G.S. Ditta, F. Vergara-Silva, M.F. Yanofsky, MADS-box gene evolution beyond flowers: expression in pollen, endosperm, guard cells, roots and trichomes, Plant J. Cell Mol. Biol. 24 (2000) 457-466.

[92] T. Hernandez-Hernandez, L.P. Martinez-Castilla, E.R. Alvarez-Buylla, Functional diversification of B MADS-box homeotic regulators of flower development: adaptive evolution in protein-protein interaction domains after major gene duplication events, Mol. Biol. Evol. 24 (2007) 465-481.

[93] L.P. Martinez-Castilla, E.R. Alvarez-Buylla, Adaptive evolution in the Arabidopsis MADS-box gene family inferred from its complete resolved phylogeny, Proc. Natl. Acad. Sci. U. S. A. 100 (2003) 13407-13412.

[94] G. Ditta, A. Pinyopich, P. Robles, S. Pelaz, M.F. Yanofsky, The SEP4 gene of Arabidopsis thaliana functions in floral organ and meristem identity, Curr. Biol. 14 (2004) 1935-1940. 
[95] S. Pelaz, R. Tapia-Lopez, E.R. Alvarez-Buylla, M.F. Yanofsky, Conversion of leaves into petals in Arabidopsis, Curr. Biol. 11 (2001) 182-184.

[96] S. Pelaz, G.S. Ditta, E. Baumann, E. Wisman, M.F. Yanofsky, B and C floral organ identity functions require SEPALLATA MADS-box genes, Nature 405 (2000) 200-203.

[97] V.F. Irish, A. Litt, Flower development and evolution: gene duplication, diversification and redeployment, Curr. Opin. Genet. Dev. 15 (2005) 454-460.

[98] M. Freeling, B.C. Thomas, Gene-balanced duplications, like tetraploidy, provide predictable drive to increase morphological complexity, Genome Res. 16 (2006) 805-814.

[99] J.A. Birchler, R.A. Veitia, The gene balance hypothesis: from classical genetics to modern genomics, Plant Cell 19 (2007) 395-402.
[100] B. Papp, C. Pal, L.D. Hurst, Dosage sensitivity and the evolution of gene families in yeast, Nature 424 (2003) 194-197.

[101] M. Bekaert, G.C. Conant, Transcriptional robustness and protein interactions are associated in yeast, BMC Syst. Biol. 5 (2011) 62.

[102] C. Diaz Arenas, T.F. Cooper, Mechanisms and selection of evolvability: experimental evidence, FEMS Microbiol. Rev. 37 (2013) 572-582.

[103] J.M. Smith, J. Haigh, The hitch-hiking effect of a favourable gene, Genet. Res 23 (1974) 23-35.

[104] O.M. Keane, C. Toft, L. Carretero-Paulet, G.W. Jones, M.A. Fares, Preservation of genetic and regulatory robustness in ancient gene duplicates of Saccharomyces cerevisiae, Genome Res. 24 (11) (2014 Nov) 1830-1841.

Please cite this article in press as: M.A. Fares, Survival and innovation: The role of mutational robustness in evolution, Biochimie (2014), http:// dx.doi.org/10.1016/j.biochi.2014.10.019 